\title{
Impacts of Absorber Loadings on Simulating the Multipath Channel in a Reverberation Chamber
}

\author{
Xin Zhou, ${ }^{1,2}$ Zhangdui Zhong, ${ }^{1}$ Xin Bian, ${ }^{2}$ Ruisi He, ${ }^{1}$ Ke Guan, ${ }^{1}$ Ruoyu Sun, ${ }^{3}$ \\ Ke Liu, ${ }^{2}$ and Xiaotao Guo ${ }^{2}$ \\ ${ }^{1}$ State Key Laboratory of Rail Traffic Control and Safety, Beijing Jiaotong University, Beijing, China \\ ${ }^{2}$ Division of Electronics and Information Technology, National Institute of Metrology, Beijing, China \\ ${ }^{3}$ Communications Technology Laboratory, National Institute of Standards and Technology, Boulder, CO, USA
}

Correspondence should be addressed to Ruisi He; ruisi.he@bjtu.edu.cn

Received 21 September 2016; Accepted 7 November 2016; Published 4 April 2017

Academic Editor: Ana Alejos

Copyright $\odot 2017$ Xin Zhou et al. This is an open access article distributed under the Creative Commons Attribution License, which permits unrestricted use, distribution, and reproduction in any medium, provided the original work is properly cited.

In reverberation chambers, the multipath channels with different delay characteristics can be generated by loading the chamber with different amounts of absorbers. This paper investigates the impacts of absorber loadings on the delay characteristics based on realistic measurements. An efficient method for estimating the root-mean-square (rms) delay spread in the chamber is presented. Furthermore, it is found that the chamber loadings also significantly affect the quality of a digitally modulated signal and the corresponding modulation quality measurements are performed.

\section{Introduction}

Reverberation chambers (RCs) are traditionally used for electromagnetic compatibility (EMC) measurements [1, 2], and during the past decade this test facility has also attracted a lot of attentions in the wireless communication field [3-5]. In such applications, the RC is used to simulate a multipath channel. The RC is basically a metal cavity that is stirred to emulate the channel [6].

The channel characteristics in the RC have been studied in many works [7-11]. For example, Genender et al. [7] showed how the power delay profile can be measured and controlled inside a RC, and it was shown that changing the loading influences the power delay profile. In $[8,9]$, root-mean-square (rms) delay spread was measured inside a RC with different amounts of absorber. The measured results were compared with those obtained in some real-world environments. The papers demonstrated that it is possible to simulate various real-world environments inside the RCs by changing the loadings. In $[10,11]$, theoretical derivation of the relationship of rms delay spread and coherence bandwidths in RC was presented. To verify the theoretical results, measurements in a RC with different loadings were conducted. All these papers showed that the channel characteristics can be adjusted by loading chamber, and some papers, for example, [8, 11], have qualitatively described the relation between the rms delay spread and the amounts of absorbing objects; that is, adding absorber will shorten the rms delay spread. However, in some applications, for example, over-the-air (OTA) test, the delay spread of the channels in the RC significantly affects the test results. Then here is one question: what is the accurate quantitative relation between the amounts of absorbers and the rms delay spread in the RC? This problem has not been well solved in the existing literatures.

The absorber loading has a significant impact on the rms delay spread of the generated channel and thus affects the signal quality. Some papers have studied the impact of absorber loading on the bit error rate (BER) $[8,12]$; however, modulation quality parameters, for example, Error Vector Magnitude (EVM), as alternative metrics offer several advantages. Firstly, measurements of EVM and related modulation quality parameters can pinpoint the type of degradations in a signal $[13,14]$. Secondly, the BER measurement relying on the Monte Carlo approach has a long measurement time, especially at low BERs [8]. By contrast, the modulation quality parameters can be readily evaluated by transmitting fewer symbols, as compared to the BER. Hence, characterizing the performance using modulation quality parameters 


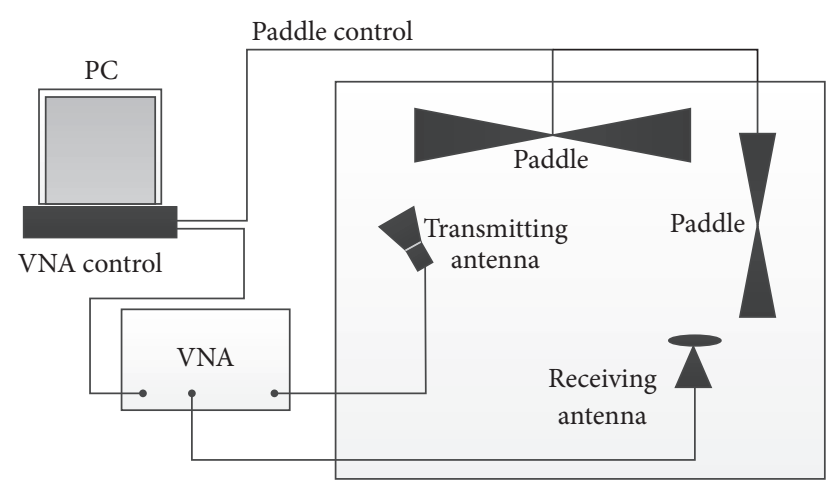

FIGURE 1: The rms delay spread measurement setup.

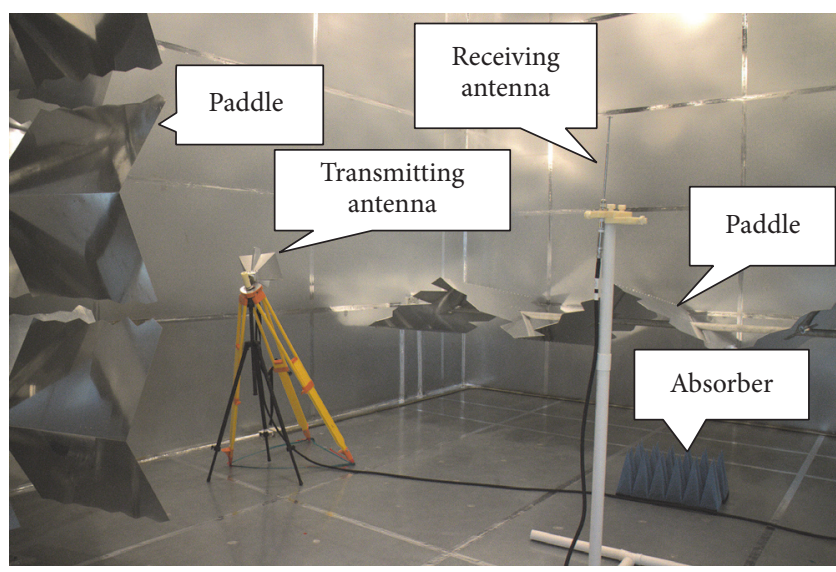

FIGURE 2: Reverberation chamber configuration.

is preferred. However to the best of the authors' knowledge, little work has been conducted to address the impact on the modulation quality parameters. This paper aims to fill this gap.

The main contributions of this paper may be summarized as follows:

(1) An efficient method to accurately estimate the rms delay spread in the RC with amounts of absorbers is presented.

(2) The influences of heavy loading on the variance of the rms delay spread in the RC are discussed.

(3) The effect of the absorber loading on the modulation quality of a digitally modulated signal is investigated.

\section{Impact on rms Delay Spread}

The rms delay spread is widely used to characterize the delay dispersion of the channel [15]. To study the effects of the absorber loading on the rms delay spread, we used the measurement system as shown in Figure 1. The test chamber was the National Institute of Metrology (NIM) reverberation chamber, having a dimension of $5.09 \mathrm{~m} \times 6.43 \mathrm{~m} \times 5.57 \mathrm{~m}$, as shown in Figure 2. The large size yields a generated channel with large rms delay spread and is suitable for replicating environments with a large number of multipath components, for example, urban environments [16]. The transmitting antenna was a horn antenna directed into a corner of the chamber, and the receiving antenna was a biconical antenna placed in the middle of the RC. The mechanical stirring was performed by two metal plates that were moved stepwise. We used two sizes of absorbers. As shown in Figure 3, for the small one, each piece is with 6 cones in a $3 \times 2$ array, the cone width is $10.2 \mathrm{~cm}$, and the cone height is $30.5 \mathrm{~cm}$. For the large one, each piece is with 81 cones in a $9 \times 9$ array, the cone width is $6.8 \mathrm{~cm}$, and the cone height is $17.8 \mathrm{~cm}$.

Measurements were conducted in the frequency domain by measuring the channel transfer function (CTF) over a frequency ranging from $4.9 \mathrm{GHz}$ to $5.1 \mathrm{GHz}$, using the vector network analyzer- (VNA-) based measurement system as shown in Figure 1 [17]. We can get the empirical channel impulse responses (CIRs), $h(\tau)$, by performing an inverse fast Fourier transform algorithm (IFFT) of the measured CTF, $H(f)$, as [17]

$$
h(\tau)=\operatorname{IFFT}(H(f))
$$

and the CIR can be expressed as

$$
h(\tau)=\sum_{i=1}^{N} a_{i} e^{-j \phi_{i}} \delta\left(\tau-\tau_{i}\right),
$$

where $a_{i}, \phi_{i}$, and $\tau_{i}$ denote the amplitude, phase, and delay of the $i$ th multipath component (MPC), respectively, $N$ is the total number of MPCs, and $\delta(\cdot)$ is the Dirac delta function. The power delay profile (PDP) of the channel is defined as the square magnitude of the CIR as follows:

$$
\operatorname{PDP}(\tau)=|h(\tau)|^{2} .
$$

Then the rms delay spread can be calculated as

$$
\tau_{\mathrm{rms}}=\sqrt{\frac{\sum_{i=1}^{N} \operatorname{PDP}\left(\tau_{i}\right) \tau_{i}^{2}}{\sum_{i=1}^{N} \operatorname{PDP}\left(\tau_{i}\right)}-\left(\frac{\sum_{i=1}^{N} \operatorname{PDP}\left(\tau_{i}\right) \tau_{i}}{\sum_{i=1}^{N} \operatorname{PDP}\left(\tau_{i}\right)}\right)^{2}},
$$

where $\tau_{i}$ and $\operatorname{PDP}\left(\tau_{i}\right)$ represent the delay and corresponding delay power of the $i$ th MPC measured, respectively. When computing the rms delay spread, we set the power of all the MPCs below a threshold to zero to reduce the impact of the noise. The threshold is set to be $6 \mathrm{~dB}$ above the noise floor [18].

The measurement was repeated for 100 different fixed paddle positions spaced by $360^{\circ} / 100=3.6^{\circ}$. The large number of paddle positions provides sufficient measurement data for the analysis of the variance of the rms delay spread. For the rms delay spread estimation, the 100 measurement results were averaged to obtain the mean values for various amounts of absorbers. To show the variance of the rms delay spread, the maximum, minimum, standard deviation (SD), and relative standard deviation (RSD, equal to $\mathrm{SD} /$ mean) of the rms delay spread are also summarized in Table 1. 


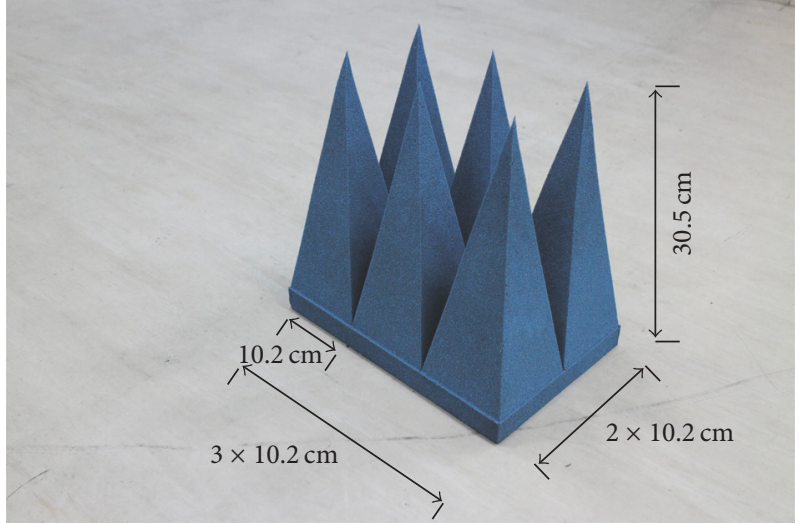

(a)

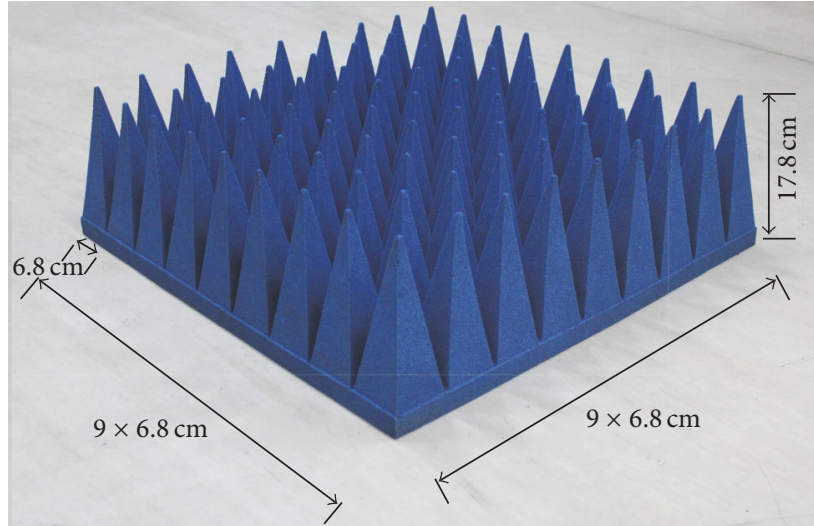

(b)

FIgURE 3: Absorbers used in our measurements. (a) Small absorber. (b) Large absorber.

TABLE 1: Statistics of rms delay spread for different numbers of absorbers.

\begin{tabular}{|c|c|c|c|c|c|c|}
\hline \multirow{2}{*}{ Number of absorbers } & \multicolumn{6}{|c|}{ RMS delay spread } \\
\hline & Mean (ns) & $\operatorname{Max}(\mathrm{ns})$ & Min (ns) & $\mathrm{SD}(\mathrm{ns})$ & RSD & error $_{r}$ \\
\hline 0 & 3050 & 3121 & 2946 & 31.11 & $1.02 \%$ & 1 \\
\hline \multicolumn{7}{|l|}{ Large absorbers } \\
\hline 1 & 1859 & 1976 & 1781 & 37.37 & $2.01 \%$ & $0 \%$ \\
\hline 2 & 1365 & 1592 & 1300 & 52.42 & $3.84 \%$ & $2.06 \%$ \\
\hline 3 & 1081 & 1323 & 977 & 72.43 & $6.70 \%$ & $3.44 \%$ \\
\hline 4 & 892 & 1128 & 789 & 74.30 & $8.33 \%$ & $4.03 \%$ \\
\hline \multicolumn{7}{|l|}{ Small absorbers } \\
\hline 1 & 2660 & 2780 & 2582 & 40.43 & $1.52 \%$ & $0 \%$ \\
\hline 2 & 2374 & 2507 & 2291 & 45.34 & $1.91 \%$ & $0.65 \%$ \\
\hline 3 & 2143 & 2307 & 2066 & 46.29 & $2.16 \%$ & $1.15 \%$ \\
\hline 4 & 1999 & 2213 & 1932 & 50.37 & $2.52 \%$ & $3.82 \%$ \\
\hline
\end{tabular}

It has been shown that the rms delay spread is directly related to the chamber quality factor by the following [8]:

$$
\tau_{\mathrm{rms}}=\frac{Q}{2 \pi f}
$$

where $f$ is the carrier frequency and the quality factor, $Q$, is the proportion of energy stored to the energy dissipated. The value of $Q$, that is, $Q_{\text {total }}$ in (6), is given by [19]

$$
Q_{\text {total }}^{-1}=Q_{\text {wall }}^{-1}+Q_{\text {antenna }}^{-1}+Q_{\text {absorber }}^{-1}
$$

where $Q_{\text {wall }}$ is associated with the wall loss, $Q_{\text {antenna }}$ is associated with energy dissipated in the antenna, and $Q_{\text {absorber }}$ is associated with absorber loss. This paper focuses on the relation between the number of absorbing objects and the generated rms delay spread. Then (6) is rewritten as

$$
Q_{\text {total }}^{-1}=Q_{\text {test_system }}^{-1}+Q_{\text {absorber } \_n}^{-1}
$$

where $Q_{\text {test_system }}^{-1}$ equals $Q_{\text {wall }}^{-1}+Q_{\text {antenna }}^{-1}$ and the subscript " $n$ " of $Q_{\text {absorber_n }}^{-1}$ indicates the number of absorbers. The combination of (5) and (7) makes it possible to relate the number of absorbers and the rms delay spread; that is,

$$
\tau_{\mathrm{rms}\left(\operatorname{total} \_n\right)}^{-1}=\tau_{\mathrm{rms}(\text { test_system })}^{-1}+\tau_{\mathrm{rms}\left(\text { absorber } \_\right)}^{-1},
$$

where $\tau_{\text {rms(total_n) }}$ is the rms delay spread for the RC with $n$ absorbers, that is, the mean values in Table $1 . \tau_{\text {rms(test_system) }}$ is the rms delay spread for the RC with unloading.

Then $\tau_{\text {rms(absorber_n) }}^{-1}\left(\right.$ equal to $\left.\tau_{\left.\text {rms(total } \_n\right)}^{-1}-\tau_{\text {rms(test_system) }}^{-1}\right)$ can be calculated from the measurement results. The calculated $\tau_{\text {rms(absorber_n) }}^{-1}$ is shown in Figure 4 . By fitting the experimental data with a least squares regression curve, a linear model is suggested:

$$
\tau_{\text {rms(absorber } \left.\_n\right)}^{-1}=n \cdot\left(\tau_{\text {rms(absorber_1 })}^{-1}\right) .
$$

The combination of (8) and (9) yields

$$
\tau_{\mathrm{rms}\left(\text { total_} \_n\right)^{-1}}^{-1}=\tau_{\mathrm{rms}(\text { test_system })^{-1}}+n \cdot\left(\tau_{\left.\mathrm{rms}_{\left(\text {absorber }_{1}\right)}^{-1}\right)},\right.
$$




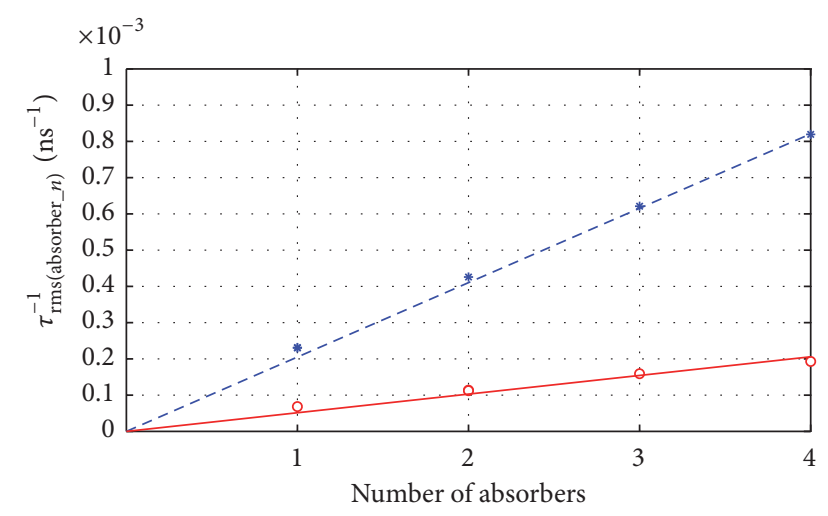

* Mean values for large loadings

- Mean values for small loadings

- - - Fitting for large loadings

_ Fitting for small loadings

FIGURE 4: $\tau_{\text {rms (absorber_n) }}^{-1}$ with different numbers of absorbers, together with the least square fit curves.

where (10), the rms delay spread for the RC (mean value) with $n$ absorbers, $\tau_{\text {rms(total } n) \text {, can be estimated. In the model }}$ of (10), $\tau_{\text {rms(test_system) }}$ and $\tau_{\text {rms(absorber_1) }}$ can be obtained from the measurements with unloading and one loading in the $\mathrm{RC}$, respectively. In our measurements, $\tau_{\text {rms(test_system) }}=$ 3050 (ns); and for the large absorber case, $\tau_{\text {rms(absorber_1 } 1)}=$ $1 /\left(\tau_{\text {rms(total_1 })}^{-1}-\tau_{\text {rms(test_system })}^{-1}\right)=4760(\mathrm{~ns})$, and for the small absorber case, $\tau_{\text {rms(absorber_1) }}=20802$ (ns).

For the proposed model of (10) applied to all the chamber loadings in Table 1, the estimated relative error for $n$ absorbers can be calculated as

$$
\operatorname{error}_{r \_n}=\frac{\left|\tau_{\text {rms }\left(\text { total } \_n\right)}-\tau_{\text {rms }\left(\text { meas } \_\right)}\right|}{\tau_{\text {rms }\left(\text { meas } \_n\right)}},
$$

where $\tau_{\operatorname{rms}(\operatorname{total} n)}$ is the estimated rms delay spread for $n$ absorbers and $\tau_{\left.\text {rms(meas } \_n\right)}$ is the measured rms delay spread, that is, the mean values in Table 1. In Table 1, error ${ }_{r}$ for different numbers of absorbers was also listed. The mean relative error is $2.52 \%$. The maximum relative error is $4.02 \%$ corresponding to the four large absorbers loaded, which is more accurate compared with other methods [19].

Furthermore, in Table 1, we find that SD and RSD increase monotonically with the increasing amount of absorbers for both the large absorbers and small absorbers. A potential explanation is that the heavy loading decreases the uniformity in the RC and leads to the variance of the rms delay spread. The decrease of uniformity also increases the measurement uncertainty. As suggested in $[20,21]$, the intrinsic field uncertainty (IFU) associated with random fields inside the $\mathrm{RC}$ is the major contribution to the overall measurement uncertainty and the IFU at receiver can be evaluated as [20]

$$
u_{\tau_{\mathrm{rms}}}=\frac{\mathrm{SD}}{\sqrt{M}},
$$

where $M$ is number of paddle positions, that is, the average number. In our measurements, the maximum $u_{\tau_{\mathrm{rms}}}$ is $7.4 \mathrm{~ns}$

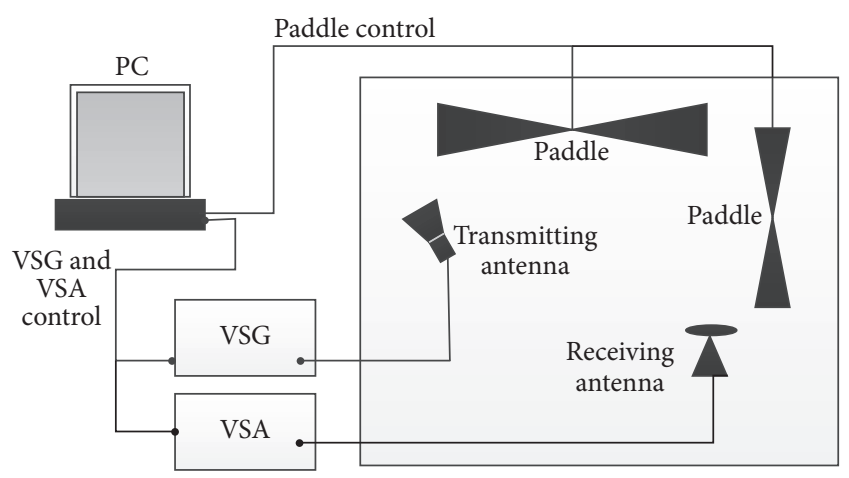

FIGURE 5: Modulation quality measurement setup.

corresponding to the four large absorbers loaded, which is much less than the measurement results (the mean values in Table 1).

\section{Impact on Modulation Quality}

In order to investigate the impact of chamber loading on the quality of digitally modulated signals, we carried out modulation quality measurements inside the RC with different loadings.

EVM is a key measure used to quantify the performance of a digital signal $[13,22]$. An ideal signal would have all constellation points precisely at the ideal locations; however various imperfections including the influence of channel characteristics cause the actual constellation points to deviate from the ideal locations. Figure 6 shows a vectorial representation using the constellation diagram. The error vector between the ideal complex-valued symbol $r(l)=r_{I}(l)+j \cdot r_{\mathrm{Q}}(l)$ and the received symbol $s(l)=s_{I}(l)+j \cdot s_{\mathrm{Q}}(l)$ is defined as $e(l)=s(l)-r(l)$. For our measurements, a total number of 1000 symbols are measured, and then $\mathrm{EVM}_{\mathrm{rms}}$ can be expressed as follows:

$$
\mathrm{EVM}_{\mathrm{rms}}=\sqrt{\frac{(1 / L) \sum_{l=1}^{L}|s(l)-r(l)|^{2}}{P_{0}}},
$$

where $P_{0}$ is average symbol power and $L$ is number of measured symbols. As one of the most important modulation quality parameters, $\mathrm{EVM}_{\mathrm{rms}}$ has been used to study the effects of the physical propagation channel on a digitally modulated signal $[14,23]$.

The measurement setup for obtaining the EVM is shown in Figure 5. A vector signal generator (VSG) was used to generate the Quadrature Phase Shift Keying (QPSK) signals with different symbol rates $(15 \mathrm{ks} / \mathrm{s}$ and $60 \mathrm{ks} / \mathrm{s})$. The output power of the VSG was $10 \mathrm{dBm}$. The center frequency of the modulated signals was $5 \mathrm{GHz}$. Up to four large absorbers were used to investigate the impact of the chamber loading on the modulation quality. A vector signal analyzer (VSA) was used to receive waveforms, demodulate them, and measure the modulation quality. Again, we placed a horn antenna directed into a corner as the transmitting antenna and a discone antenna in the middle of the RC as the receiving 
TABLE 2: Statistics of $\mathrm{EVM}_{\mathrm{rms}}$ for different numbers of absorbers.

\begin{tabular}{|c|c|c|c|c|c|c|}
\hline \multirow{2}{*}{ Symbol rate } & \multirow{2}{*}{ Number of absorbers } & \multicolumn{5}{|c|}{$\mathrm{EVM}_{\mathrm{rms}}$} \\
\hline & & Mean (\%) & $\operatorname{Max}(\%)$ & $\operatorname{Min}(\%)$ & $\mathrm{SD}(\%)$ & RSD \\
\hline \multirow{5}{*}{$15 \mathrm{ks} / \mathrm{s}$} & 0 & 8.24 & 42.81 & 1.14 & 8.57 & $104 \%$ \\
\hline & 1 & 4.66 & 35.73 & 1.26 & 5.77 & $124 \%$ \\
\hline & 2 & 3.59 & 30.85 & 1.21 & 3.77 & $105 \%$ \\
\hline & 3 & 3.37 & 16.31 & 1.18 & 2.57 & $76 \%$ \\
\hline & 4 & 3.05 & 26.59 & 1.24 & 3.02 & $99 \%$ \\
\hline \multirow{5}{*}{$60 \mathrm{ks} / \mathrm{s}$} & 0 & 20.61 & 49.60 & 4.66 & 11.36 & $55 \%$ \\
\hline & 1 & 12.76 & 48.73 & 2.67 & 10.13 & $79 \%$ \\
\hline & 2 & 9.07 & 39.38 & 1.81 & 6.85 & $75 \%$ \\
\hline & 3 & 8.49 & 45.28 & 2.53 & 7.44 & $87 \%$ \\
\hline & 4 & 7.01 & 47.79 & 2.07 & 6.12 & $87 \%$ \\
\hline
\end{tabular}

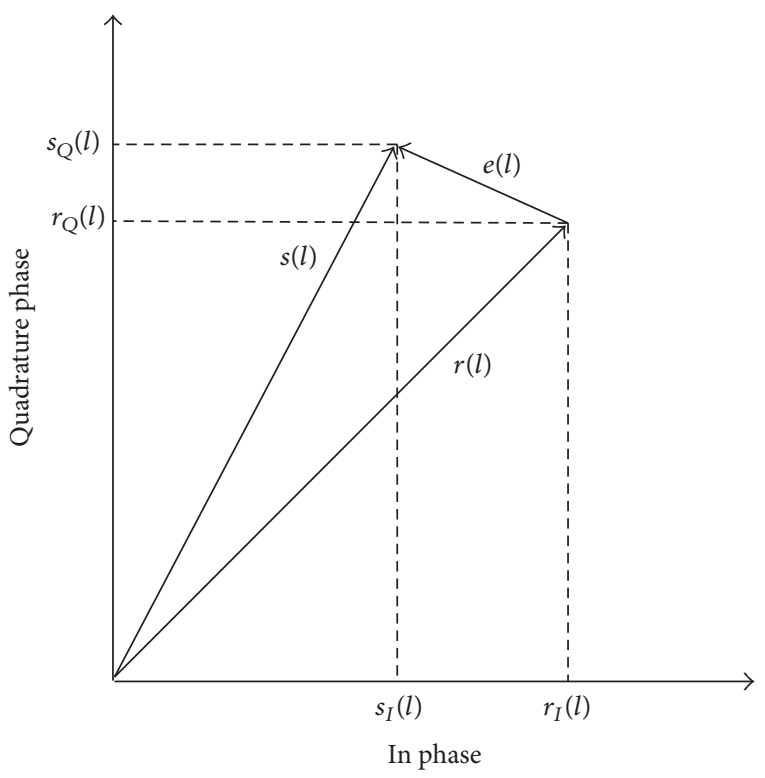

FIGURE 6: Constellation diagram and EVM.

antenna. The $\mathrm{EVM}_{\mathrm{rms}}$ measurement was also repeated for 100 different fixed paddle positions as the rms delay spread measurements.

Before conducting the measurements in the RC, modulation quality measurements were performed with connecting the VSA and VSG directly with a cable, where $\mathrm{EVM}_{\mathrm{rms}}$ is found to be $0.25 \%$ and $0.27 \%$ for $15 \mathrm{ks} / \mathrm{s}$ and $60 \mathrm{ks} / \mathrm{s}$, respectively. The low residual errors enable the study of RCinduced effects rather than receiver-induced effects on the modulation quality.

The measurement results of the mean values of $\mathrm{EVM}_{\mathrm{rms}}$ are shown in Figure 7. Firstly, it can be seen that low symbol rate leads to a better modulation quality. The lower symbol rate essentially implies a narrower bandwidth, which is less sensitive to frequency selectivity of channel. This is the reason that the signal with lower symbol rate will degrade less for given absorbers. Furthermore, as shown in Figure 7, heavy loading leads to better modulation quality. As mentioned

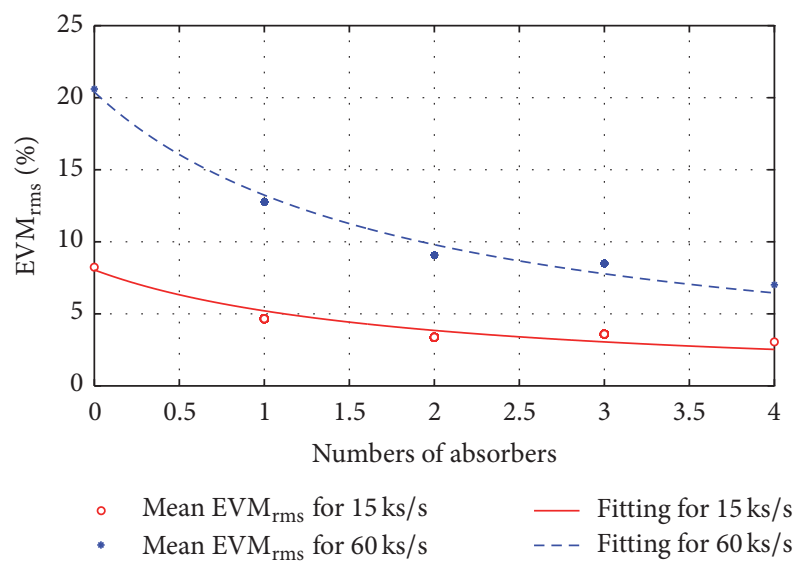

FIgURE 7: The mean values of $\mathrm{EVM}_{\mathrm{rms}}$ with different numbers of absorbers, together with the least square fit curves.

above, heavy loading leads to smaller delay spread. It has been shown that the coherence bandwidth (as the measure of the frequency selectivity) is reversely proportional to the rms delay spread $[10,11]$. Therefore, adding absorber will widen the coherence bandwidth and finally leads to better modulation quality [12].

As observed in Figure 7, the mean value of $\mathrm{EVM}_{\mathrm{rms}}$ fits to a rational function well, which can be written as

$$
\mathrm{EVM}_{\mathrm{rms}}=\frac{p}{n+q},
$$

where $n$ indicates the number of absorbers. $p$ and $q$ are the coefficients for the rational function. For the symbol rate of $15 \mathrm{ks} / \mathrm{s}, p=0.15$ and $q=1.82$; for the symbol rate of $60 \mathrm{ks} / \mathrm{s}, p=0.38$ and $q=1.85$. Note that this model is heuristic, whose ultimate justification lies in its ability to fit the measurement data.

Table 2 shows the statistics of $\mathrm{EVM}_{\mathrm{rms}}$. It is found that the SD and RSD are considerably high. The reason is that $\mathrm{EVM}_{\mathrm{rms}}$ values at some paddle positions are much higher than the mean values; for example, see column Max in 


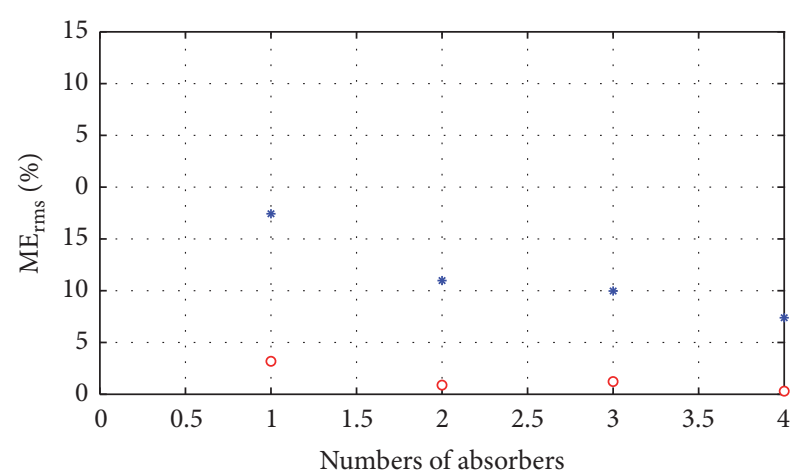

- Measurement for $15 \mathrm{ks} / \mathrm{s}$

* Measurement for $60 \mathrm{ks} / \mathrm{s}$

(a)

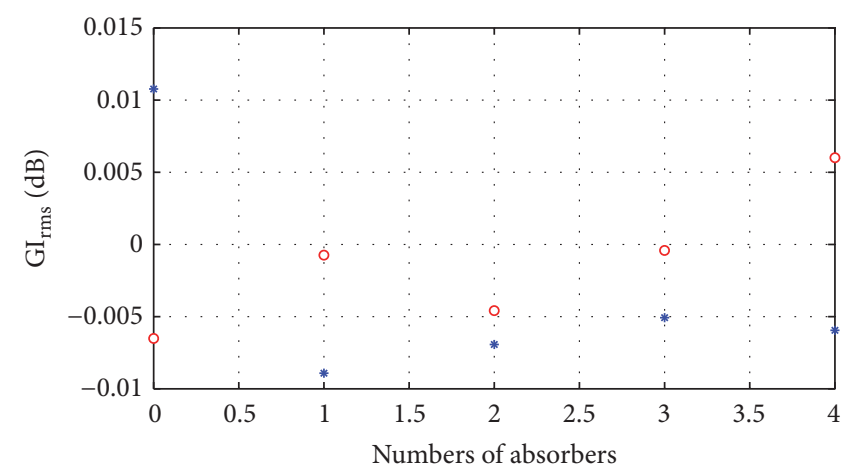

- Measurement for $15 \mathrm{ks} / \mathrm{s}$

* Measurement for $60 \mathrm{ks} / \mathrm{s}$

(c)

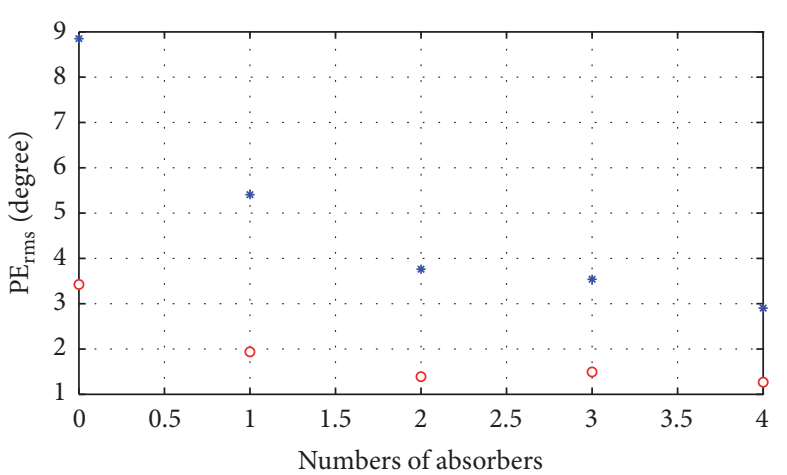

- Measurement for $15 \mathrm{ks} / \mathrm{s}$

* Measurement for $60 \mathrm{ks} / \mathrm{s}$

(b)

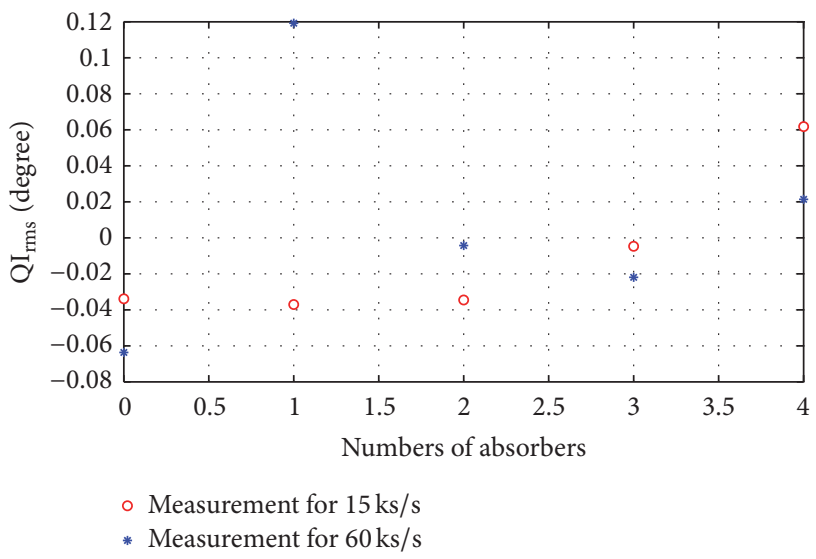

(d)

FIGURE 8: Scatter plots of the mean values of the modulation quality parameters. (a) Magnitude error. (b) Phase error. (c) Gain imbalance. (d) Quadrature imbalance.

Table 2. The high $\mathrm{EVM}_{\mathrm{rms}}$ values may come from the fact that the reflected signals cause the receiver to experience a severe fading at some paddle positions. The modulation quality of the digitally modulated signals in the RC is sensitive to the paddle positions.

The EVM is the total error and contains various degradations [13]. In order to pinpoint the degradations, four main modulation quality parameters, that is, magnitude error (ME), phase error (PE), gain imbalance (GI), and quadrature imbalance (QI) $[13,14]$, were measured with the same measurement setup as the EVM measurement. Figure 8 shows the mean values over the 100 paddle positions of these modulation quality parameters for different loadings. It is found that heavy loading decreases the magnitude error and phase error but the quadrature imbalance and gain imbalance do not decrease monotonically with the amount of absorbers.

\section{Conclusions}

This paper investigates the impacts of the absorber loadings on the delay spread and modulation quality in the RC. Based on the measurements, an efficient method for estimating the rms delay spread in the RC with the amounts of absorbing objects is presented. In addition, it is found that heavy loading leads to an increase of the variance of the rms delay spread in the chamber.

The chamber loadings are found to affect the quality of the digitally modulated signals due to different channel conditions of the generated multipath channels. The modulation quality measurements show that heavy loading leads to a better modulation quality and the modulation quality is sensitive to the paddle positions.

This paper presents implementation-specific details necessary for the use of RCs. Our analysis is based on realistic measurements, which makes the conclusion sufficiently reliable and can be applied when using a RC to simulate wireless propagation environments.

\section{Conflicts of Interest}

The authors declare that there is no conflict of interests regarding the publication of this paper. 


\section{Acknowledgments}

This work was supported in part by the National Key Technology R\&D Program under Grants 2014BAK02B05 and 2014BAK02B04; by the Natural Science Base Research Plan in Shanxi Province of China under Grant 2015JM6320; by National Natural Science Foundation of China under Grant 61501020; by State Key Laboratory of Rail Traffic Control and Safety under Grants RCS2016ZJ005 and RCS2015ZZ001; by China Postdoctoral Science Foundation under Grant 2016M591355; by Fundamental Research Funds for the Central Universities under Grant 2016JBZ006; and by the National 863 Key Project under Grant 2014AA01A706. The authors would like to thank Dr. Kate A. Remley and Qiulai Gao for their helpful suggestions and support. The authors also thank S. Ding and Y. Liu for their assistance during the preparation of the measurement campaign.

\section{References}

[1] C. L. Holloway, D. A. Hill, M. Sandroni et al., "Use of reverberation chambers to determine the shielding effectiveness of physically small, electrically large enclosures and cavities," IEEE Transactions on Electromagnetic Compatibility, vol. 50, no. 4, pp. 770-782, 2008.

[2] C. L. Holloway, D. A. Hill, J. Ladbury, G. Koepke, and R. Garzia, "Shielding effectiveness measurements of materials using nested reverberation chambers," IEEE Transactions on Electromagnetic Compatibility, vol. 45, no. 2, pp. 350-356, 2003.

[3] X. Chen, B. P. Einarsson, and P.-S. Kildal, "Improved MIMO throughput with inverse power allocation-study using USRP measurement in reverberation chamber," IEEE Antennas \& Wireless Propagation Letters, vol. 13, no. 13, pp. 1494-1496, 2014.

[4] R. Recanatini, F. Moglie, and V. M. Primiani, "Performance and immunity evaluation of complete wlan systems in a large reverberation chamber," IEEE Transactions on Electromagnetic Compatibility, vol. 55, no. 5, pp. 806-815, 2013.

[5] S. J. Floris, K. A. Remley, and C. L. Holloway, "Bit error rate measurements in reverberation chambers using real-time vector receivers," IEEE Antennas and Wireless Propagation Letters, vol. 9, no. 1, pp. 619-622, 2010.

[6] P. Corona, G. Ferrara, and M. Migliaccio, "Reverberating chamber electromagnetic field in presence of an unstirred component," IEEE Transactions on Electromagnetic Compatibility, vol. 42, no. 2, pp. 111-115, 2000.

[7] E. Genender, C. L. Holloway, K. A. Remley, J. Ladbury, G. Koepke, and H. Garbe, "Use of reverberation chamber to simulate the power delay profile of a wireless environment," in Proceedings of the IEEE International Symposium on Electromagnetic Compatibility (EMC '08), pp. 1-6, September 2008.

[8] E. Genender, C. L. Holloway, K. A. Remley, J. M. Ladbury, G. Koepke, and H. Garbe, "Simulating the multipath channel with a reverberation chamber: application to bit error rate measurements," IEEE Transactions on Electromagnetic Compatibility, vol. 52, no. 4, pp. 766-777, 2010.

[9] S. Herbert, T.-H. Loh, I. Wassell, and J. Rigelsford, "On the analogy between vehicle and vehicle-like cavities with reverberation chambers," IEEE Transactions on Antennas and Propagation, vol. 62, no. 12, pp. 6236-6245, 2014.

[10] X. Chen, P.-S. Kildal, C. Orlenius, and J. Carlsson, "Channel sounding of loaded reverberation chamber for over-the-air testing of wireless devices: coherence bandwidth versus average mode bandwidth and delay spread," IEEE Antennas and Wireless Propagation Letters, vol. 8, pp. 678-681, 2009.

[11] X. Chen and P.-S. Kildal, “Theoretical derivation and measurements of the relationship between coherence bandwidth and RMS delay spread in reverberation chamber," in Proceedings of the 3rd European Conference on Antennas and Propagation (EuCAP '09), pp. 2687-2690, Berlin, Germany, March 2009.

[12] K. A. Remley, S. J. Floris, H. A. Shah, and C. L. Holloway, "Static and dynamic propagation-channel impairments in reverberation chambers," IEEE Transactions on Electromagnetic Compatibility, vol. 53, no. 3, pp. 589-599, 2011.

[13] J. L. Pinto and I. Darwazeh, "Error vector magnitude relation to magnitude and phase distortion in 8-PSK systems," Electronics Letters, vol. 37, no. 7, pp. 437-438, 2001.

[14] V. A. Thomas, S. Kumar, S. Kalyani, and M. El-Hajjar, "Error vector magnitude analysis of fading SIMO channels relying on MRC reception," IEEE Transactions on Communications, vol. 64, no. 4, pp. 1786-1797, 2016.

[15] P. A. Bello, "Characterization of randomly time-variant linear channels," IEEE Transactions on Communications Systems, vol. 11, no. 4, pp. 360-393, 1963.

[16] J. Laurila, K. Kalliola, M. Toeltsch, K. Hugl, P. Vainikainen, and E. Bonek, "Wideband 3D characterization of mobile radio channels in urban environment," IEEE Transactions on Antennas \& Propagation, vol. 50, no. 2, pp. 233-243, 2002.

[17] X. Zhou, Z. Zhong, B. Zhang et al., "Experimental characterization and correlation analysis of indoor channels at $15 \mathrm{GHz}$," International Journal of Antennas and Propagation, vol. 2015, Article ID 601835, 11 pages, 2015.

[18] A. F. Molisch and M. Steinbauer, "Condensed parameters for characterizing wideband mobile radio channels," International Journal of Wireless Information Networks, vol. 6, no. 3, pp. 133$154,1999$.

[19] D. A. Hill, M. T. Ma, A. R. Ondrejka, B. F. Riddle, M. L. Crawford, and R. T. Johnk, "Aperture excitation of electrically large, lossy cavities," IEEE Transactions on Electromagnetic Compatibility, vol. 36, no. 3, pp. 169-178, 1994.

[20] IEC, "Electromagnetic compatibility (EMC)-part 4-21: testing and measurement techniques-reverberation chamber test methods," Tech. Rep. IEC 61000-4-21, 2003.

[21] L. R. Arnaut, "Measurement uncertainty in reverberation chambers-I. Sample statistics," NPL Report TQE 2, National Physical Laboratory, Teddington, UK, 2008.

[22] A. Georgiadis, "Gain, phase imbalance, and phase noise effects on error vector magnitude," IEEE Transactions on Vehicular Technology, vol. 53, no. 2, pp. 443-449, 2004.

[23] C. Jastrow, S. Priebe, B. Spitschan et al., "Wireless digital data transmission at $300 \mathrm{GHz}$," Electronics Letters, vol. 46, no. 9, pp. 661-663, 2010. 


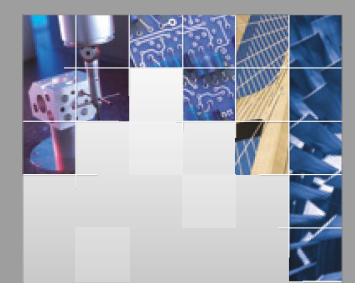

\section{Enfincering}
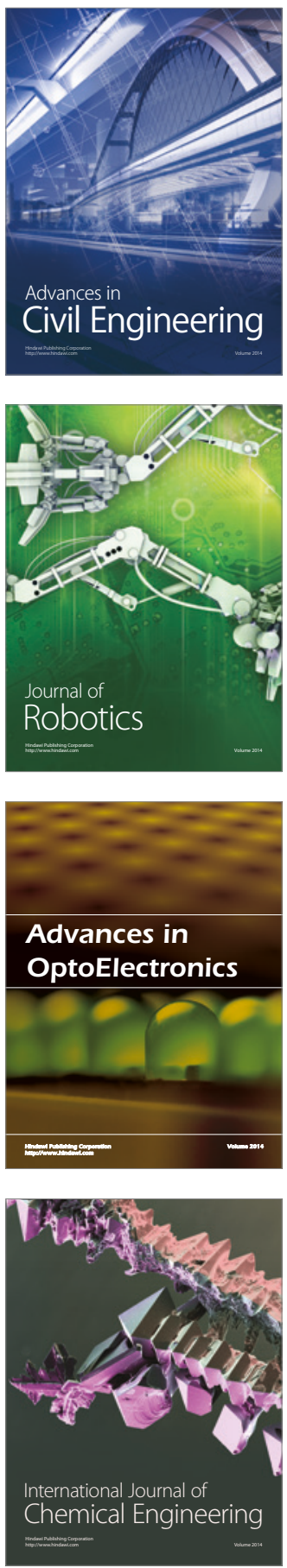

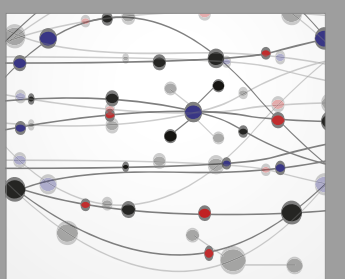

The Scientific World Journal

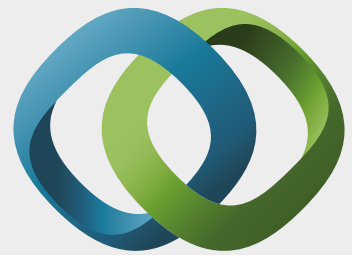

\section{Hindawi}

Submit your manuscripts at

https://www.hindawi.com
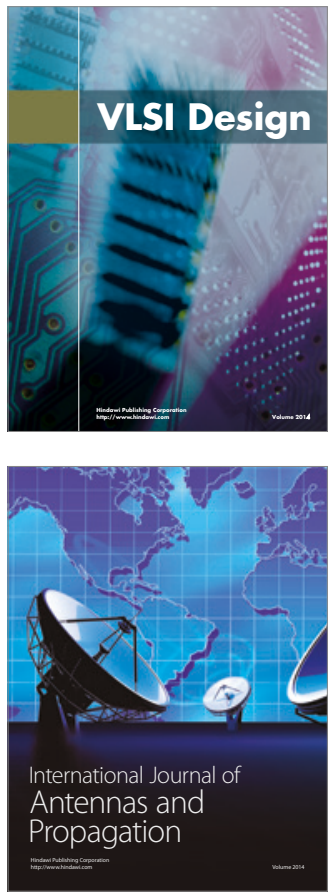

\section{Rotating}

Machinery
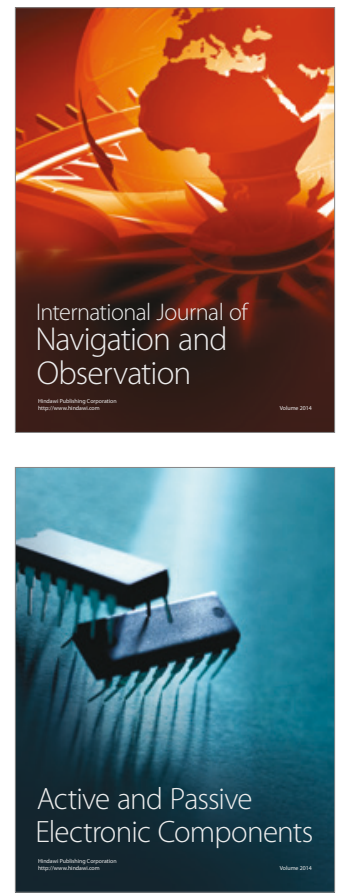
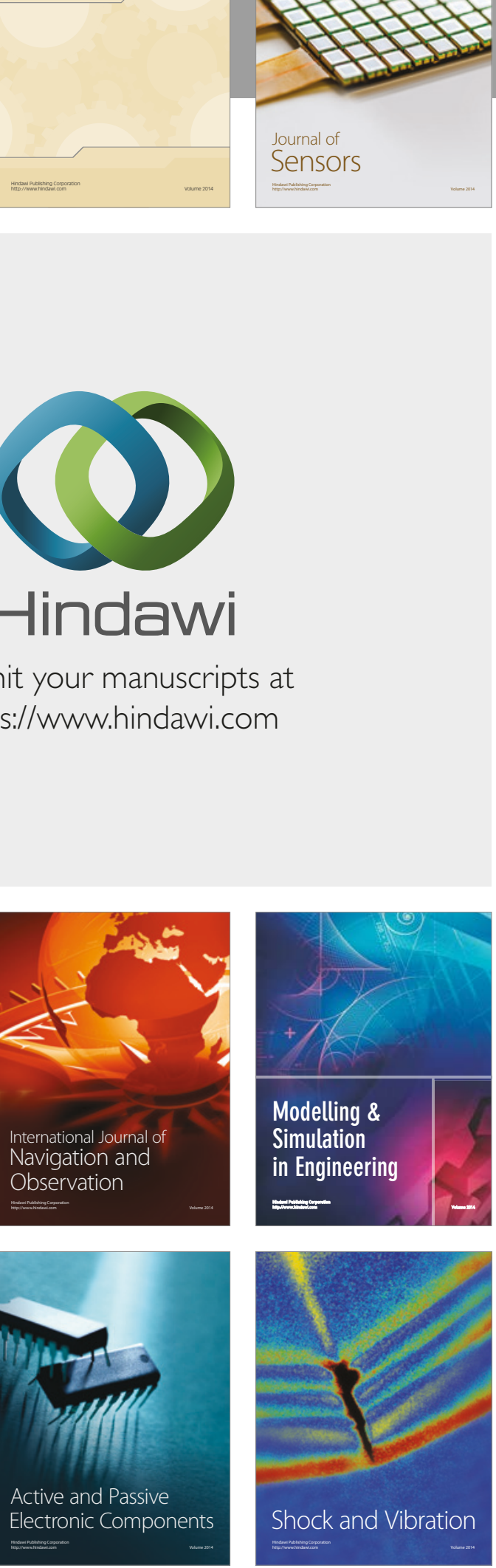
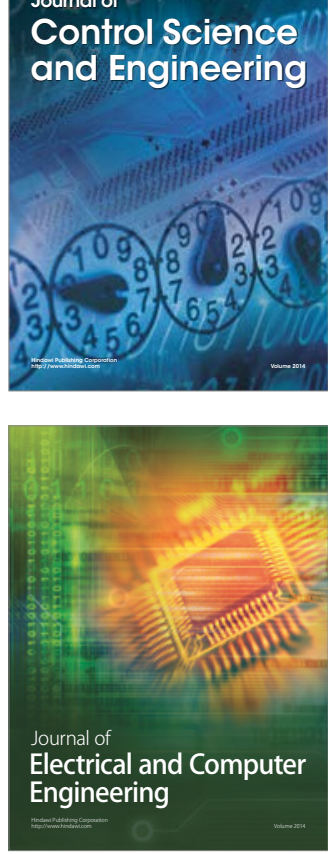

Distributed

Journal of

Control Science

and Engineering
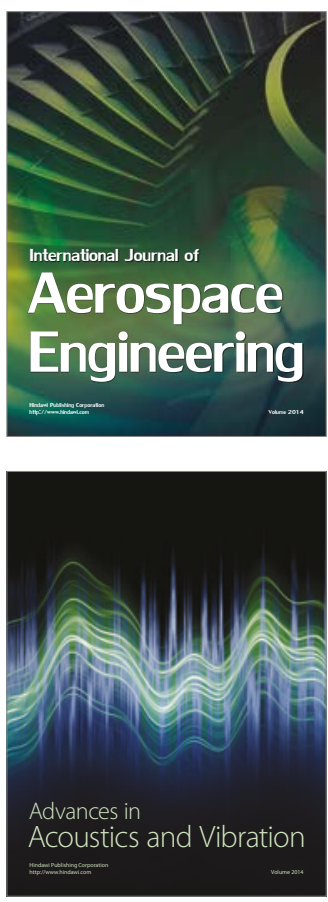

Sensor Networks 